\title{
SINGULAR f-SUM RULE FOR SUPERFLUID ${ }^{4} \mathrm{He}$
}

\author{
Victor K. WONG \\ Physics Laboratory, Department of Natural Sciences, University of Michigan-Dearborn, Dearborn, MI 48128, USA 1 \\ and Department of Physics, University of California Berkeley, CA 94720, USA
}

Received 27 June 1979

The validity and applicability to inelastic neutron scattering of a singular f-sum rule for superfluid helium, proposed by Griffin to explain the $\rho_{\mathrm{S}}$ dependence in $S(k, \omega)$ as observed by Woods and Svensson, are examined in the light of similar sum rules rigorously derived for anharmonic crystals and Bose liquids. It is concluded that the singular f-sum rules are only of microscopic interest.

Considerable interest has been generated by the recent measurements of Woods and Svensson [1] of the inelastic neutron scattering cross section of superfluid ${ }^{4} \mathrm{He}$ for temperatures $T$ from $1.0 \mathrm{~K}$ to $4.2 \mathrm{~K}$. They found that the dynamic structure function $S_{\mathrm{n}}(k, \omega)$ as a function of $T$, wavevector $k$, and frequency $\omega / 2 \pi$, is well described by a two-component form:

$S(k, \omega)=\left(\rho_{\mathrm{s}} / \rho\right) S_{\mathrm{s}}(k, \omega)+\left(\rho_{\mathrm{n}} / \rho\right) S_{\mathrm{n}}(k, \omega)$,

where the superfluid component $S_{\mathrm{s}}(k, \omega)$, has a onephonon peak characteristic of superfluid helium near $T=0$ and the normal component $S_{\mathrm{n}}(k, \omega)$ has a shape characteristic of nonsuperfluid helium. Here $\rho_{\mathrm{s}}(T)$ and $\rho_{\mathrm{n}}(T) \equiv \rho-\rho_{\mathrm{s}}(T)$ are, respectively, the usual superfluid and normal-fluid mass densities.

In an attempt to place this surprising result (1) onto a firm theoretical framework, Griffin [2] considered the singular $\mathrm{f}$-sum rule, which is a mathematical construct that gives the exact contribution to the first $\omega$ moment of

$\widetilde{S}(k, \omega)=-(1 / \pi) \operatorname{Im} \widetilde{F}(k, \omega)$,

where the density-density response $\widetilde{F}(k, \omega)$ is the total contribution from all singular diagrams, i.e., those with an isolated one-particle line. Specifically, Griffin (see eq. (12) in ref. [2]) proposed that the singular f-sum rule for superfluid helium at $T>0$ has the form

1 Permanent address. $\int \mathrm{d} \omega \omega \tilde{S}(k, \omega)=\left(\rho_{\mathrm{s}}(T) / \rho\right) k^{2} / 2 m$,

in the limit of $k \rightarrow 0$. Eq. (3) was first written down intuitively by Pines [3] and should be contrasted with the well known f-sum rule

$\int \mathrm{d} \omega \omega S(k, \omega)=k^{2} / 2 m$,

which is valid for all $k$. Furthermore, Griffin identified the singular $\widetilde{S}(k, \omega)$ in eq. (3) with the experimental $S_{\mathrm{s}}(k, \omega)$ in eq. (1).

Because the proposed singular $f$-sum rule (3) has important consequences for the interpretation of neutron scattering experiments, we examine in this note the problem of the identification of $\widetilde{S}(k, \omega)$ with a part of the experimental $S(k, \omega)$, i.e., the applicability to inelastic neutron scattering of the left-hand side of eq. (3), as well as the explicit form of the singular $f-$ sum rule for superfluid helium, i.e., the validity of the right-hand side of eq. (3). This is done by appeal to singular f-sum rules rigorously derived for anharmonic crystals and for Bose liquids.

Let us begin with the question of the identification of $\widetilde{S}(k, \omega)$ with a part of the experimental $S(k, \omega)$, as it appears for solid helium. The singular $f$-sum rule for anharmonic crystals [4] was derived by standard fieldtheoretic methods and found to have the form for all $\boldsymbol{k}$ :

$\int \mathrm{d} \omega \omega \tilde{S}(k, \omega)=\mathrm{e}^{-2 W(k)} k^{2} / 2 m$, 
where $W(k)$ is the Debye-Waller exponent. It is tempting to suppose [5] that, by separating the experimental $S(k, \omega)$ into a one-phonon peak and a smooth background and taking the ratio of the respective first moments, we can from eqs. (4) and (5) determine the Debye-Waller factor. Such an analysis, however, when performed on neutron scattering data for bcc ${ }^{4} \mathrm{He}$ [6] led to serious difficulties. On the basis of detailed calculations, Horner [7] has concluded that the experimental one-phonon contribution can not be identified with the singular $\widetilde{S}(k, \omega)$ in eq. (5).

This startling conclusion can be understood as follows. The general singular diagram that contributes to $\widetilde{F}(k, \omega)$ has the structure of two complicated vertices with an isolated one-phonon line connecting them. From eq. (2) the imaginary part of $\widetilde{F}(k, \omega)$ picks up, in addition to the resonant peak from the one-phonon line, significant nonresonant wings from the vertices. Although $S(k, \omega)$ can be shown to be positive definite, there are no grounds to suppose that $\widetilde{S}(k, \omega)$ is everywhere positive. In fact, due to interference processes, $\widetilde{S}(k, \omega)$ becomes negative within the nonresonant wings. Since $\widetilde{S}(k, \omega)$ need not be positive and does become negative, there is no a priori operational rule for separating the singular $\widetilde{S}(k, \omega)$ from the wings of the experimental $S(k, \omega)$.

This discussion for solid helium can be straightforwardly transferred (apart from polarization vectors) to superfluid helium, since the elementary excitation in both cases is given by the one-phonon state [8]. We conclude that in general the singular $\widetilde{S}(k, \omega)$ can not be identified with the one-phonon peak of the experimental $S(k, \omega)$ because of significant and as yet inextricable contributions from the wings.

The above conclusion may have gone too far, since with $\widetilde{S}(k, \omega)$ not being identified with the experimental $S_{\mathrm{s}}(k, \omega)$, the observed coefficient $\rho_{\mathrm{s}} / \rho$ in eq. (1) is clearly inconsistent with the proposed singular f-sum rule (3). The obvious next step is to consider the validity of the right-hand side of eq. (3).

A basic assumption made by Griffin [2] to justify the proposed sum rule (3) is that the vertex and selfenergy functions are nonsingular functions of $k$ and $\omega$. This assumption can be tested by calculations for the Bose gas. If the interaction between the onephonon states is negligible, the assumption is obviously true. If the interaction is not negligible, then the simpplest nontrivial structure of the vertices and self-ener- gies is that of a one-loop diagram $[9,10]$. The resulting functions are no longer nonsingular but have branch cuts. Consequences of the one-loop structure and the concomitant branch cuts include (inextricable) wings in $S(k, \omega)$ for a Bose gas [11]. Hence serious doubts are cast on eq. (3).

To replace eq. (3), we need a rigorous derivation, analogous to that in ref. [4] for anharmonic crystals, of the singular f-sum rule for Bose liquids. Such a derivation was accomplished [10] by standard field-theoretic methods. (Since this sum rule was incidental to ref. [10], it was relegated with no comments to appendix $D$ and can easily escape notice.) Although the derivation was for a Bose liquid at $T=0$, extension to $T>0$ is straightforward and gives the form for all $k$ :

$$
\begin{aligned}
& \int \mathrm{d} \omega \omega \tilde{S}(k, \omega) \\
& \quad=\left(n_{0} / n\right)\left[k^{2} / 2 m+M_{11}^{\mathrm{HF}}(k)-M_{12}^{\mathrm{HF}}(k)-\mu\right],
\end{aligned}
$$

where $n_{0}$ is the condensate number density, $n=\rho / m$, $\mu$ is the chemical potential, and $M_{i j}^{\mathrm{HF}}(k)$ is the HartreeFock matrix self-energy.

Comparing eqs. (3) with (6), we see that the significant change, besides the lifting of the $k \rightarrow 0$ limit, is the replacement of $\rho_{\mathrm{s}}$ with $n_{0}$. This replacement implies that the singular f-sum rule is a microscopic sum rule (one that measures the microscopic density $n_{0}$ rather than the macroscopic density $\rho_{\mathrm{s}}$ ), and it underscores the profound difference in a Bose liquid between $n_{0}$ and $\rho_{s}$. Comparing eqs. (5) with (6), we see that the Debye-Waller factor $\mathrm{e}^{-2 W}$ and the condensate density $n_{0}$ play analogous roles. Both quantities furnish the microscopic link between density fluctuations and the (displacement or field) amplitude fluctuations.

Until the microscopic effects on $S(k, \omega)$ are understood to the extent that the singular $\widetilde{S}(k, \omega)$ can be identified, the singular f-sum rules (5) and (6) are only of microscopic interest and remain, regrettably, not very useful for the interpretation of the surprising and still unexplained result (1). A study of eq. (1) not based on the singular $\mathrm{f}$-sum rule will be published elsewhere [12].

\section{References}

[1] A.D.B. Woods and E. Svensson, Phys. Rev. Lett. 41 (1978) 974

[2] A. Griffin, Phys. Lett. 71A (1979) 237. 
[3] D. Pines, in: Low temperature physics LT 9, eds. J.G. Daunt, D.O. Edwards, F.J. Milford and M. Yaqub (Plenum, New York, 1965) p. 61.

[4] V. Ambegaokar, J.M. Conway and G. Baym, in: Lattice dynamics, ed. R.F. Wallis (Pergamon, London, 1965) p. 261.

[5] N.R. Werthamer, Phys. Rev. Lett. 28 (1972) 1102.

[6] E.B. Osgood, V.J. Minikiewicz, T.A. Kitchens and G. Shirane, Phys. Rev. A5 (1972) 1537.
[7] H. Horner, Phys. Rev. Lett. 29 (1972) 556.

[8] V.K. Wong, J. Low Temp. Phys. 18 (1975) 65.

[9] S. Ma, H. Gould and V.K. Wong, Phys. Rev. A3 (1971) 1453.

[10] V.K. Wong and H. Gould, Ann. Phys. (NY) 83 (1974) 252.

[11] D.L. Bartley and V.K. Wong, Phys. Rev. B12 (1975) 3775.

[12] V.K. Wong, to be published. 